\title{
Single-slice Spiral Computed Tomography
}

National Cancer Institute

\section{Source}

National Cancer Institute. Single-slice Spiral Computed Tomography. NCI Thesaurus. Code C106567.

A type of spiral computed tomography that employs only one detector that produces one image (slice). 